\title{
Analysis of influencing factors related to elevated serum troponin I level for COVID-19 patients in Yichang, China
}

\author{
Zhi-Xing Fan ${ }^{1,2,3,4 \#}$, Jun Yang ${ }^{1,2,3}$, Jing Zhang ${ }^{1,2,3 \#}$, Chao $\mathrm{He}^{1,2,3}$, Hui Wu ${ }^{1,2,3}$, Chao-Jun Yang ${ }^{1,2,3 \#}$, \\ Tao Zheng ${ }^{1,2,3}$, Cong Ma ${ }^{1,2,3}$, Zu-Jin Xiang ${ }^{1,2,3}$, Yu-Hong Zhai ${ }^{1,2,3}$, Jing Jiang ${ }^{5}$, Shao-Qin Qiu ${ }^{6}$, Jian Yang ${ }^{7}$ \\ ${ }^{1}$ Department of Cardiology, ${ }^{2}$ Central Laboratory, the First College of Clinical Medical Sciences, China Three Gorges University \& Yichang Central \\ People's Hospital, Yichang, China; ${ }^{3}$ Yichang Key Laboratory of Ischemic Cardiovascular and Cerebrovascular Disease Translational Medicine, \\ Yichang, China; ${ }^{4}$ Department of Cardiology, Renmin Hospital of Wuhan University, Wuhan, China; ${ }^{5}$ Yichang Center for Disease Control and \\ Prevention, Yichang, China; ${ }^{6}$ Department of Cardiology, Yichang Third People's Hospital, Yichang, China; ${ }^{7}$ Department of Cardiology, the People's \\ Hospital of Three Gorges University \& the First People's Hospital of Yichang, Yichang, China \\ Contributions: (I) Conception and design: ZX Fan, J Zhang, J Yang, SQ Qiu; (II) Administrative support: ZX Fan, CJ Yang, J Yang; (III) Provision of \\ study materials or patients: C He, H Wu, J Jiang, C Ma; (IV) Collection and assembly of data: ZJ Xiang, YH Zhai, T Zheng, C Ma; (V) Data analysis \\ and interpretation: CJ Yang, ZX Fan, C Ma; (VI) Manuscript writing: All authors; (VII) Final approval of manuscript: All authors. \\ "These authors contributed equally to this work. \\ Correspondence to: Jian Yang. Department of Cardiology, the People's Hospital of Three Gorges University \& the First People's Hospital of Yichang, \\ 4 Hudi Street, Yichang, China. Email: yangjian@ctgu.edu.cn; Jun Yang. Department of Cardiology, the First College of Clinical Medical Sciences, \\ China Three Gorges University \& Yichang Central People’s Hospital, 183 Yilin Road, Yichang, China. Email: yangjun@ctgu.edu.cn.
}

Background: Cardiac injury is a common condition among hospitalized coronavirus disease 2019 (COVID-19) patients, and is associated with a higher risk of mortality. However, the mechanism of myocardial injury in COVID-19 remains unclear. In this retrospective study, we compared the clinical characteristics of COVID-19 patients with different troponin I (TnI) levels during hospitalization to provide a clinical reference for the identification of those at high-risk.

Methods: In total, 218 patients diagnosed with COVID-19 in Yichang Central People's Hospital and Yichang Third People's Hospital between January 23 and February 19, 2020 were initially included. Of these patients, 89 underwent TnI testing during hospitalization and were finally included in the study. The medical history, clinical signs and symptoms at the time of admission, and laboratory test results were recorded. The patients were assigned to the normal $\mathrm{TnI}$ group $(\mathrm{TnI}<0.01 \mu \mathrm{g} / \mathrm{L} ; \mathrm{n}=67$ ) or the elevated $\mathrm{TnI}$ group (TnI $>0.01 \mu \mathrm{g} / \mathrm{L} ; \mathrm{n}=22)$.

Results: The incidence of elevated TnI in our patient cohort was $24.7 \%$. There were significant differences between the two groups in the following factors: history of coronary heart disease (CHD), age, lymphocyte count, prothrombin time (PT), activated partial thromboplastin time (APTT), and levels of interleukin (IL)6, C-reactive protein (CRP), myoglobin (MYO), lactate dehydrogenase $(\mathrm{LDH})$, and albumin (all $\mathrm{P}<0.05)$. Binary logistic analysis showed that a history of CHD, age, lymphocyte count, IL-6, APTT, and MYO were influencing factors of elevated serum TnI.

Conclusions: A history of CHD, advanced age, decreased lymphocyte count, increased IL-6, increased MYO, and prolonged APTT were independent influencing factors of elevated TnI in COVID-19 patients. COVID-19 patients with these characteristics are prone to myocardial injury.

Keywords: Coronavirus disease 2019 patients (COVID-19 patients); myocardial injury; troponin I (TnI); influencing factors

Submitted May 17, 2020. Accepted for publication Jul 09, 2020.

doi: $10.21037 / \mathrm{cdt}-20-510$

View this article at: http://dx.doi.org/10.21037/cdt-20-510 


\section{Introduction}

The novel coronavirus pneumonia caused by the SARSCoV-2 pathogen has been officially named coronavirus disease 2019 (COVID-19) by the World Health Organization (1). Since its outbreak in 2019, this pathogen has caused a worldwide pandemic. As of June 25, 2020, there have been more than 9.4 million people diagnosed with COVID-19 and more than 470,000 deaths worldwide. In-depth observations and studies have shown that some COVID-19 patients have clinical manifestations of cardiac involvement in addition to the typical respiratory symptoms; this cardiac involvement greatly accelerates the development of the disease and increases the difficulty of treating these patients (2). Numerous studies have shown that COVID-19 patients with myocardial injury are prone to cardiac dysfunction and malignant arrhythmia, with a significantly shortened survival time and a significantly increased risk of in-hospital death $(3,4)$.

The mechanism of myocardial injury related to COVID-19 is unclear, and there are few studies on the factors that influence myocardial injury in these patients (5). Myocardial injury in COVID-19 patients appears to be associated with the disease prognosis. Therefore, a detailed analysis of the influencing factors in such patients could help to establish an effective disease early warning monitoring system to guide early treatment measures and minimize death caused by myocardial injury.

The most typical clinical feature of myocardial injury related to COVID-19 is the presence of elevated myocardial injury markers that exceed the upper limit of the 99th percentile in the absence of clinical evidence of myocardial ischemia (3). Elevated troponin levels are associated with poor prognosis and high mortality in many diseases $(6,7)$, and troponin I (TnI) is the most sensitive and specific marker for assessing myocardial injury. In this study, we compared the differences in clinical features of COVID-19 patients categorized by TnI level to explore factors that could be used to provide a clinical reference for identifying high-risk patients. We present the following article in accordance with the STROBE reporting checklist (available at http://dx.doi.org/10.21037/cdt-20-510).

\section{Methods}

\section{Subjects}

COVID-19 patients who were admitted and diagnosed in Yichang Central People's Hospital and Yichang Third
People's Hospital between January 23, 2020 and February 19, 2020 were screened continuously. According to the interim guidance of the World Health Organization (8), all included patients had a positive SARS-CoV-2 nucleic acid detection result assessed by real-time fluorescence RT-PCR. We used serum TnI levels to divide patients into a normal group $(\mathrm{TnI}<0.01 \mu \mathrm{g} / \mathrm{L})$ and an elevated group (TnI $>0.01 \mu \mathrm{g} / \mathrm{L})$. Patients were excluded if they had medical history of an acute heart-related condition such as acute myocardial infarction, rheumatic heart disease (acute phase), or cardiac surgery within 1 month. The study was conducted in accordance with the Declaration of Helsinki (as revised in 2013). This study was approved by the ethics committee of Yichang Central People's Hospital and Yichang Third People's Hospital (No. 20200413001). This investigation was a retrospective study, and patients were exempted from providing informed consent.

\section{Collection of clinical data}

The clinical data of the study patients were collected through the electronic medical record system and included the following: (I) general information including sex, age, and smoking history; (II) previous medical history of hypertension, coronary heart disease (CHD), diabetes, chronic lung disease, chronic kidney disease, cerebrovascular disease, or malignant tumor; (III) main clinical manifestations at the time of admission including fever, cough, sore throat, dyspnea, fatigue, muscle aches, palpitations, chest tightness, chest pain, headache, and diarrhea; (IV) highest body temperature before admission, heart rate at admission, and respiratory rate at admission; and (V) results of laboratory tests taken 24 hours after admission [white blood cell (WBC), neutrophil, lymphocyte, monocyte, platelet counts; levels of hemoglobin, interleukin (IL)-6, procalcitonin (PCT), C-reactive protein (CRP), D-dimer, prothrombin time (PT), activated partial thromboplastin time (APTT), creatine kinase (CK), CK$\mathrm{MB}$ isozyme, myoglobin (MYO), lactate dehydrogenase (LDH), hydroxybutyrate dehydrogenase (HBDH), alanine aminotransferase (ALT), aspartate aminotransferase (AST), creatinine $(\mathrm{Cr})$, glycosylated hemoglobin (HbA1c), lowdensity lipoprotein cholesterol (LDL-C), high-density lipoprotein cholesterol (HDL-C), N-terminal pro hormone $\mathrm{B}$-type natriuretic peptide (NT-proBNP), albumin, globulin, albumin/globulin ratio (A/G), and $\mathrm{CO}_{2}$ binding capacity]; and (VI) complications including acute respiratory distress syndrome (ARDS), acute kidney injury, malignant 
arrhythmia, and shock.

\section{Statistical analysis}

The statistical processing of all data was performed using SPSS version 25.0 (IBM). Measurement data with a normal distribution and homogeneity of variance are expressed as the mean \pm standard deviation $(\bar{x} \pm s)$, and an independent sample $t$-test was used for comparisons between groups. Measurement data with a non-normal distribution are expressed as the median $\left(25^{\text {th }}\right.$ percentile, $75^{\text {th }}$ percentile), and the Mann-Whitney $U$ test was used for comparisons between groups. Count data are expressed as percentages, and the $\chi^{2}$ test or Fisher's exact test was used for comparisons between groups. Multivariate analysis was performed using binary logistic regression. The R3.6.1 software nomogram package was used to establish a predictive nomogram. $\mathrm{P}<0.05$ was considered to be statistically significant.

\section{Results}

\section{Medical history}

A total of 218 patients were initially included according to the COVID-19 diagnostic criteria. Among them, eightynine of these patients had undergone TnI testing during hospitalization and were ultimately included in this study (40.8\%); 67 were assigned to the normal group and 22 to the elevated group. The incidence of elevated $\mathrm{TnI}$ in this COVID-19 patient cohort was $24.7 \%$. There were no significant differences between the normal TnI group and the elevated TnI group in sex, fever, cough, sore throat, fatigue, muscle aches, palpitations, chest pain or tightness, dyspnea, headache, diarrhea, smoking history, history of hypertension, diabetes, chronic lung disease, chronic kidney disease, cerebrovascular disease and malignancy, heart rate at admission, respiratory rate at admission, or highest body temperature before admission (all $\mathrm{P}>0.05$ ). There were significant differences in the history of CHD and age between the two groups (both $\mathrm{P}<0.05$ ). These results are presented in Table 1.

\section{Laboratory test results}

There were no significant differences between the normal and elevated $\mathrm{TnI}$ groups in any of the following factors: counts of WBCs, neutrophils, monocytes, platelets; levels of hemoglobin, PCT, D-dimer, CK, CK-MB, HBDH,
ALT, AST, Cr, HbA1c, LDL-C, HDL-C, NT-proBNP, and globulin; $\mathrm{A} / \mathrm{G}$ ratio; or $\mathrm{CO}_{2}$ binding capacity (all $\mathrm{P}>0.05$ ). There were significant differences in the lymphocyte count, levels of IL-6, CRP, PT, APTT, MYO, LDH, and albumin between the two groups (all $\mathrm{P}<0.05$ ). These results are presented in Table 2.

\section{Binary logistic regression analysis of factors influencing elevated TnI in COVID-19 patients}

The initial modeling included a history of CHD, age, lymphocyte count, and levels of IL-6, CRP, PT, APTT, MYO, LDH, and albumin. After excluding irrelevant factors and considering tolerance, the final modeling results showed that a history of CHD, advanced age, decreased lymphocyte count, increased levels of IL-6 and MYO, and prolonged APTT were independent influencing factors of elevated TnI, as shown in Table 3. The results suggested that the incidence of elevated $\mathrm{TnI}$ is significantly increased in older COVID-19 patients who have a history of CHD, a decreased lymphocyte count, increased IL-6 and MYO levels, and prolonged APTT.

\section{Effect of elevated TnI on complications in COVID-19 patients}

About a quarter $(24.7 \%)$ of the COVID-19 patients in our cohort had an elevated TnI level. Compared with patients with normal TnI, patients with elevated TnI had significantly higher incidences of complications including ARDS, acute kidney injury, and malignant arrhythmia during hospitalization (all $\mathrm{P}<0.05$ ). These results are presented in Table 4.

\section{Predictive nomogram for TnI elevation}

The results of binary logistic regression analysis (i.e., history of CHD, age, lymphocyte count, IL-6 and MYO levels, and APTT) were used to establish a nomogram model to predict the elevation of TnI (Figure 1). This figure shows the points corresponding to the different values of each variable, and the individual total score is calculated accordingly to provide the probability of $\mathrm{TnI}$ elevation for COVID-19 patients.

\section{Discussion}

Studies have shown that some patients with COVID-19 
Table 1 Medical history of patients in the two groups

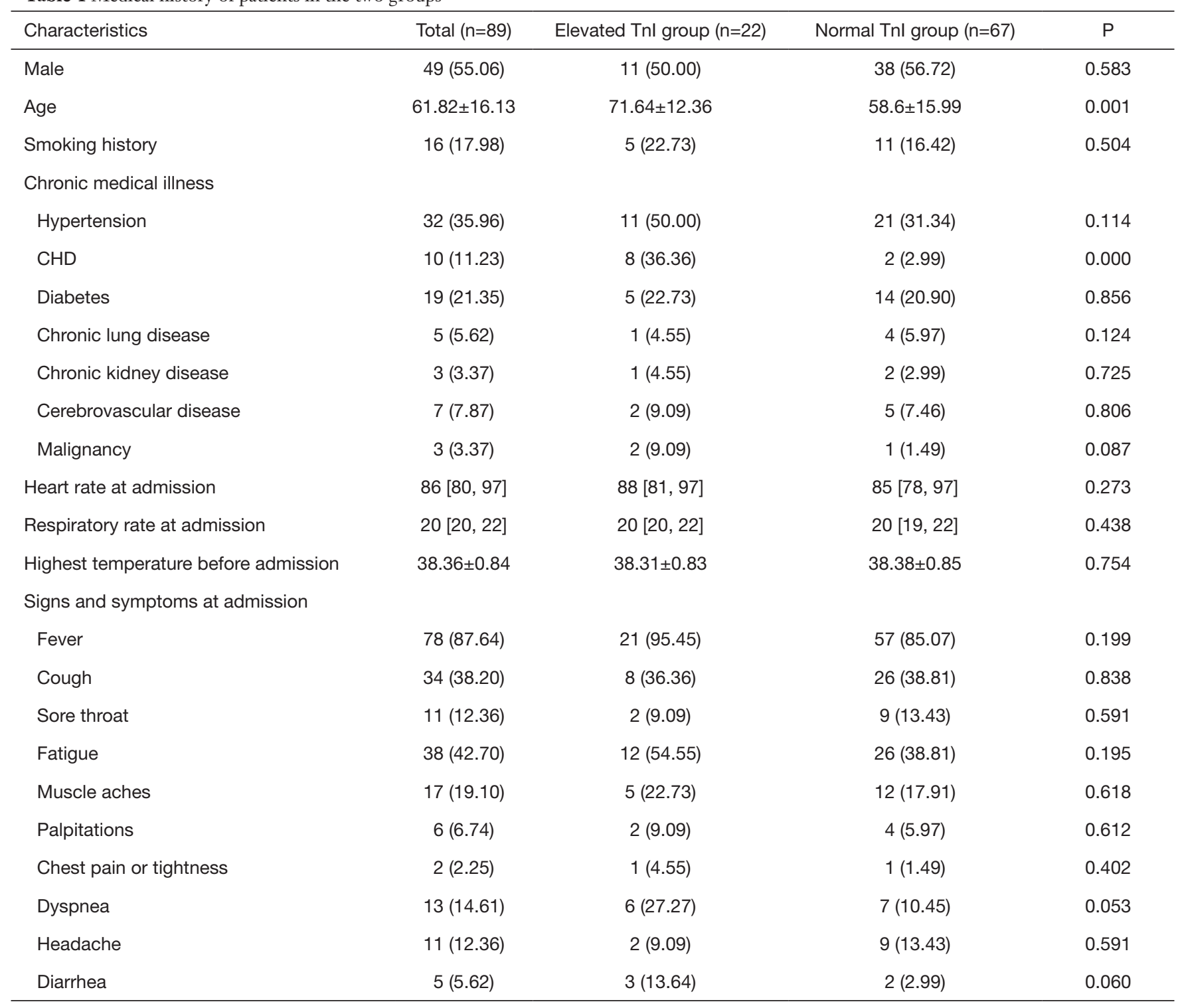

Data are present as $\bar{x} \pm s, \mathrm{M}[\mathrm{P} 25, \mathrm{P} 75]$ or $\mathrm{n}(\%) . \mathrm{Tnl}$, troponin I; CHD, coronary heart disease.

have troponin levels that increase as the disease progresses, and this trend is associated with a poor prognosis $(3,4,9,10)$. Huang et al. reported that among the first 41 patients to be diagnosed with COVID-19 in Wuhan, 5 (12\%) were diagnosed with acute myocardial injury, with the main manifestation being elevated TnI (9). Shi et al. investigated 416 COVID-19 patients and found that $19.7 \%$ had elevated TnI levels during hospitalization; $51.2 \%$ of those died; whereas the patients without elevated TnI levels had a mortality rate of only $4.5 \%$ (3). Guo et al. analyzed the clinical data of 187 patients with COVID-19 and found that
$27.8 \%$ experienced myocardial injury during the course of the disease and were prone to cardiac dysfunction and malignant arrhythmia (4). Therefore, it can be concluded that the patient's TnI level can be used to evaluate prognosis in COVID-19. Furthermore, our thorough analysis of the factors influencing myocardial injury in COVID-19 patients will help to establish an effective early warning monitoring system to initiate early treatment and minimize the risk of death caused by myocardial injury.

In this study, we found that $24.7 \%$ of patients with COVID-19 had elevated TnI levels, but their disease 
Table 2 Laboratory test results in the two groups

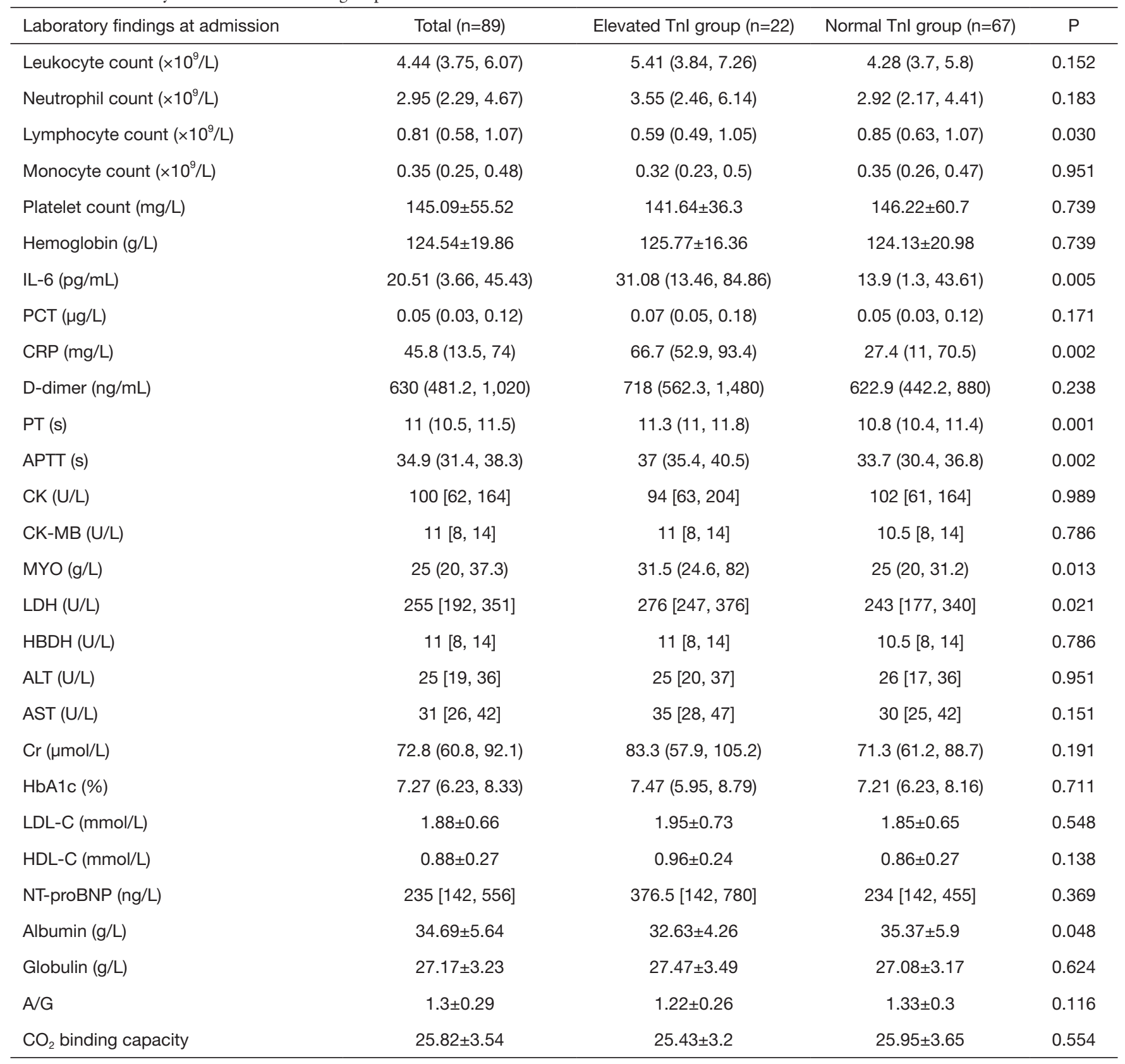

Tnl, troponin I; IL-6, interleukin 6; PCT, procalcitonin; CRP, C-reactive protein; PT, prothrombin time; APTT, activated partial thromboplastin time; CK, creatine kinase; CK-MB, creatine kinase isoenzyme; MYO, myoglobin; LDH, lactate dehydrogenase; HBDH, hydroxybutyrate dehydrogenase; ALT, alanine aminotransferase; AST, aspartate aminotransferase; Cr, creatinine; HbA1c, glycated hemoglobin; LDL-C, low density lipoprotein cholesterol; HDL-C, high density lipoprotein cholesterol; NT-proBNP, N-terminal brain natriuretic peptide precursor; A/G, albumin/globulin ratio.

symptoms and signs were not specific. Among the patients with elevated TnI, there was a high incidence of complications including ARDS, acute kidney injury, and malignant arrhythmia. A history of CHD, advanced age, decreased lymphocyte count, increased levels of IL-6 and $\mathrm{MYO}$, and prolonged APTT were shown to be independent influencing factors of elevated $\mathrm{TnI}$ and can be used as the main predictive factors for myocardial injury. Therefore, 
Table 3 Binary logistic regression analysis of influencing factors related to elevated TnI in COVID-19 patients

\begin{tabular}{lccccc}
\hline Characteristic & B & S.E & Wals & P & Exp (B) \\
\hline Age & -0.084 & 0.032 & 6.997 & 0.008 & 0.919 \\
CHD & -1.967 & 0.976 & 4.062 & 0.044 & 0.14 \\
Lymphocyte count $\left(\times 10^{9} / \mathrm{L}\right)$ & 2.366 & 1.182 & 4.008 & 0.045 & 10.658 \\
IL-6 (pg/mL) & -0.034 & 0.012 & 7.298 & 0.007 & 0.967 \\
MYO (g/L) & -0.006 & 0.002 & 6.485 & 0.011 & 0.994 \\
APTT (s) & -0.163 & 0.076 & 4.58 & 0.032 & 0.849 \\
Constant & 12.633 & 3.853 & 10.751 & 0.001 & $306,589.472$ \\
\hline
\end{tabular}

Tnl, troponin I; CHD, coronary heart disease; IL-6, interleukin 6; MYO, myoglobin; APTT, activated partial thromboplastin time.

Table 4 Effect of elevated TnI on the complications of COVID-19 patients

\begin{tabular}{lcccc}
\hline Complications & Total $(\mathrm{n}=89)$ & Elevated Tnl group $(\mathrm{n}=22)$ & Normal Tnl group $(\mathrm{n}=67)$ & $\mathrm{P}$ \\
\hline ARDS & $32(35.96)$ & $12(54.55)$ & $20(29.85)$ & 0.036 \\
Acute kidney injury & $19(21.35)$ & $10(45.45)$ & $9(13.43)$ & 0.001 \\
Malignant arrhythmia & $6(6.74)$ & $4(18.18)$ & $2(2.99)$ & 0.014 \\
Shock & $13(14.61)$ & $5(22.73)$ & $8(11.94)$ & 0.214 \\
\hline
\end{tabular}

Data are present as $\mathrm{n}(\%)$. Tnl, troponin I; ARDS, acute respiratory distress syndrome.

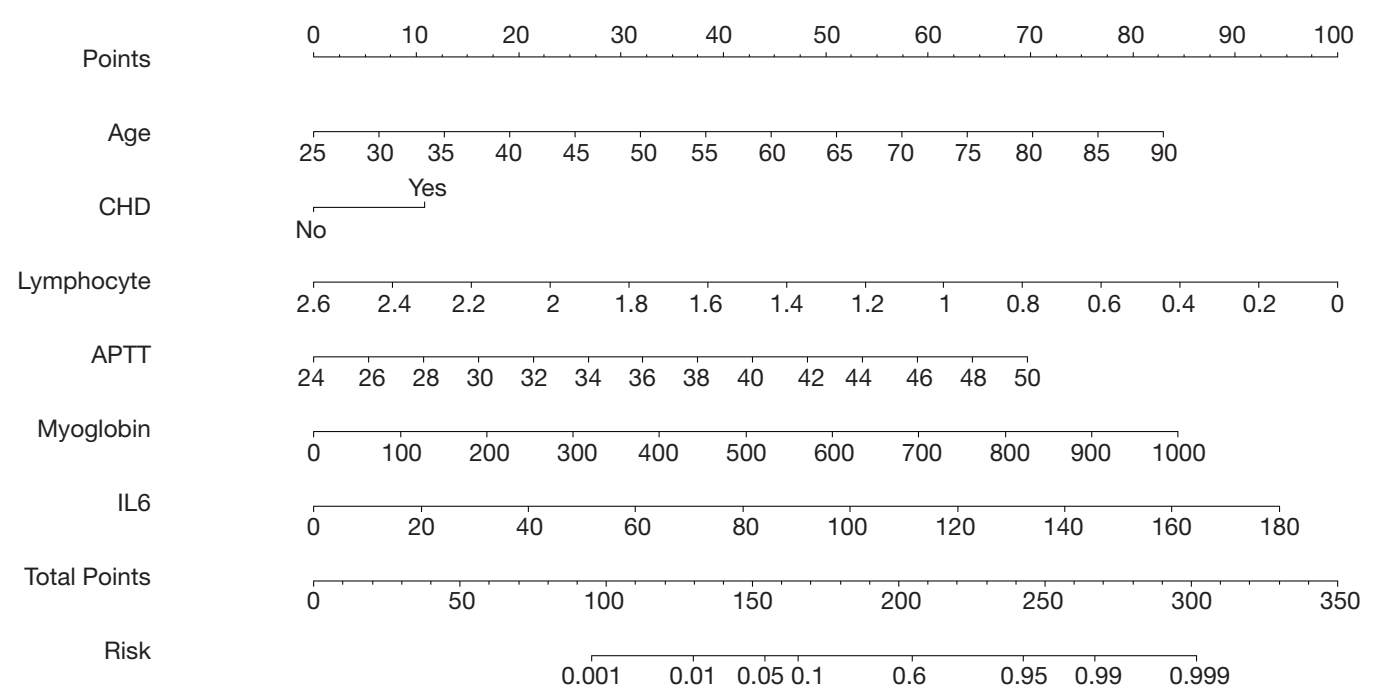

Figure 1 The predictive nomogram for TnI elevation. TnI, troponin I; CHD, coronary heart disease; APTT, activated partial thromboplastin time; IL-6, interleukin 6.

it is especially important to monitor older COVID-19 patients with CHD for their lymphocyte count, IL-6 and MYO levels, and APTT. A nomogram model was also drawn to provide a more intuitive tool to predict elevated
TnI.

According to the existing epidemiological data, older patients with CHD who become infected with COVID-19 seem to have disproportionately high rates of critical illness 
and mortality, but the specific mechanisms for this remain unclear (11-13). In the current study, older COVID-19 patients with $\mathrm{CHD}$ were prone to having elevated $\mathrm{TnI}$ levels, suggesting that the poor prognosis in these patients may be related to TnI. One explanation for this phenomenon may be the difference in albumin levels between the normal and elevated TnI groups. This finding suggested a tendency for older patients to have poor nutritional status and decreased autoimmune function, which can lead to delayed recovery or even accelerated progression of COVID-19 (14). Furthermore, COVID-19-induced stress may cause decreased plaque stability, reduced oxygen supply and increased oxygen consumption, thereby aggravating myocardium ischemia and increasing the risk of coronary events (15).

Similar to SARS-CoV, SARS-CoV-2 invades human respiratory epithelia via binding of the viral S-protein to its cell-surface receptor-angiotensin converting enzyme 2 (ACE2) (16). ACE2 can also show high expression on the surface of cardiovascular endothelial cells; therefore, it is speculated that SARS-CoV-2 may damage the myocardium by binding to highly expressed ACE2 in the heart tissue $(16,17)$. However, recent pathological studies have shown that interstitial infiltration of mononuclear inflammatory factors is rare in the myocardial tissues of patients with COVID-19, which indicates that SARS-CoV-2 may not damage the heart directly (18). Further analysis showed that the number of $\mathrm{CD}^{+}$and $\mathrm{CD} 8^{+}$cells in the peripheral blood was greatly reduced in COVID-19 patients, but that their immune cell status was highly activated. Upon activation, the large numbers of inflammatory cytokines that are rapidly produced and can create a "cytokine storm", causing immune injury to cardiomyocytes and eventually leading to circulatory failure in COVID-19 patients (18). The results of the current study showed that a decreased lymphocyte count and increased IL-6 levels were closely related to elevated TnI, confirming that excessive immune activation can cause myocardial injury in COVID-19 patients. It should be noted that, CRP, another inflammatory marker, showed a difference in the univariate analysis, but was not found to be independently related to elevated $\mathrm{TnI}$ in the multivariate logistic regression analysis. We speculate that increased CRP levels are caused by multiple factors in COVID-19 patients.

The acute inflammatory response to infection can affect coagulation and fibrinolysis through multiple pathways, eventually leading to disorder in the coagulation cascade and fibrinolysis processes, thereby promoting microthrombosis (19).
The formation of microthrombi can lead to myocardial injury as a result of an imbalance in oxygen supply and demand (20). In this study, the differences in PT and APTT between patients in the elevated and normal TnI groups were statistically significant, and multivariate logistic regression analysis showed that APTT was an independent risk factor for elevated $\mathrm{TnI}$.

We found that the proportions of COVID-19 patients with increased levels of elevated LDH and MYO were higher in the elevated-TnI group, with multivariate logistic regression analysis showing an independent association between MYO and elevated TnI. MYO is found in skeletal muscle and the myocardium and is a sensitive and specific biomarker for the diagnosis of myocardial infarction and CHD in the acute phase. After myocardial injury, the rise in serum MYO occurs earlier than the rise in TnI (21), possibly explaining why MYO was independently related to elevated TnI in this study. LDH can be found in almost all tissue types (22). However, we did not find an independent correlation between increased LDH and TnI levels, which, like CRP, might have been due to the effects of multiple other factors on LDH.

This study has some limitations. First, the patients were not followed up to evaluate the long-term prognostic value of TnI level in COVID-19. Second, potentially important clinical data such as echocardiography, cardiac color Doppler ultrasound, treatment protocol, and clinical outcomes were not collected. Third, although our patients came from two hospitals that were designated for the treatment of COVID-19 in Yichang, Hubei Province, the sample size was small, and may have led to bias in the statistical analysis. Finally, Yichang lies outside of Wuhan; therefore, COVID-19 patients in this study may have comprised mainly second or third generation cases. Hence, the virulence of the virus may have been weakened and the degree of TnI elevation may be different compared with earlier patients from Wuhan. Therefore, the results presented here need to be verified and confirmed by largescale multicenter studies.

\section{Conclusions}

A history of CHD, advanced age, decreased lymphocyte count, increased IL-6, increased levels of MYO and prolonged APTT were identified as independent influencing factors of elevated TnI. Clinicians should consider the possibility of myocardial injury in such COVID-19 patients and provide appropriate cardiovascular-specific diagnosis 
and treatment to save lives.

\section{Acknowledgments}

The authors thank all the front-line workers in the fight against coronavirus disease 2019.

Funding: This work was supported by the National Natural Science Foundation of China (Grant No. 81800258; 81770360; 81670333), Hubei Province Health and Family Planning Scientific Research Project (WJ2019Z004), Hubei Provincial Natural Science Foundation of China (2018CFA044), Hubei Province's Outstanding Medical Academic Leader program, and the Medical and Health Research Project of Yichang city, China (Grant No. A20-2004).

\section{Footnote}

Reporting Checklist: The authors present the study in accordance with the STROBE reporting checklist. Available at http://dx.doi.org/10.21037/cdt-20-510

Data Sharing Statement: Available at http://dx.doi. org/10.21037/cdt-20-510

Peer Review File: Available at http://dx.doi.org/10.21037/ cdt-20-510

Conflicts of Interest: All authors have completed the ICMJE uniform disclosure form (available at http://dx.doi. org/10.21037/cdt-20-510). The authors have no conflicts of interest to declare.

Ethical Statement: The authors are accountable for all aspects of the work in ensuring that questions related to the accuracy or integrity of any part of the work are appropriately investigated and resolved. The study was conducted in accordance with the Declaration of Helsinki (as revised in 2013). The study was approved by ethics committee of Yichang Central People's Hospital and Yichang Third People's Hospital (No. 20200413001). This investigation was a retrospective study, and patients were exempted from providing informed consent.

Open Access Statement: This is an Open Access article distributed in accordance with the Creative Commons Attribution-NonCommercial-NoDerivs 4.0 International License (CC BY-NC-ND 4.0), which permits the non- commercial replication and distribution of the article with the strict proviso that no changes or edits are made and the original work is properly cited (including links to both the formal publication through the relevant DOI and the license). See: https://creativecommons.org/licenses/by-nc-nd/4.0/.

\section{References}

1. Lai CC, Shih TP, Ko WC, et al. Severe acute respiratory syndrome coronavirus 2 (SARS-CoV-2) and coronavirus disease-2019 (COVID-19): the epidemic and the challenges. Int J Antimicrob Agents 2020;55:105924.

2. Wang D, Hu B, Hu C, et al. Clinical characteristics of 138 hospitalized patients with 2019 novel coronavirus-infected pneumonia in Wuhan, China. JAMA 2020;323:1061-9.

3. Shi S, Qin $M$, Shen B, et al. Association of cardiac injury with mortality in hospitalized patients with COVID-19 in Wuhan, China. JAMA Cardiol 2020;5:802-10.

4. Guo T, Fan Y, Chen M, et al. Cardiovascular implications of fatal outcomes of patients with coronavirus disease 2019 (COVID-19). JAMA Cardiol 2020;5:1-8.

5. Madjid M, Solomon S, Vardeny O. ACC clinical bulletin: cardiac implications of novel Wuhan coronavirus (2019$\mathrm{nCoV}) .2020$. Available online: https://www.acc.org/latestin-cardiology/articles/2020/02/13/12/42/acc-clinicalbulletin-focuses-on-cardiac-implications-of-coronavirus2019-ncov

6. Wu CM, Hu XL, Song JX, et al. Heart injury signs are associated with higher and earlier mortality in coronavirus disease 2019 (COVID-19). medRxiv preprint. 2020. doi: https://doi.org/10.1101/2020.02.26.20028589.

7. Stacy SR, Suarez-Cuervo C, Berger Z, et al. Role of troponin in patients with chronic kidney disease and suspected acute coronary syndrome: a systematic review. Ann Intern Med 2014;161:502-12.

8. World Health Organization. Clinical management of severe acute respiratory infection when novel coronavirus $(\mathrm{nCoV})$ infection is suspected: interim guidance. 2020. Available online: https://www.who.int/publicationsdetail/clinical-management-of-severe-acute-respiratoryinfection-when-novel-coronavirus-(ncov)-infection-issuspected

9. Huang C, Wang Y, Li X, et al. Clinical features of patients infected with 2019 novel coronavirus in Wuhan, China. Lancet 2020;395:497-506.

10. Shi S, Qin M, Cai YL, et al. Characteristics and clinical significance of myocardial injury in patients with severe coronavirus disease 2019. Eur Heart J 2020;41:2070-9. 
11. Epidemiology Working Group for NCIP Epidemic Response, Chinese Center for Disease Control and Prevention. The epidemiological characteristics of an outbreak of 2019 novel coronavirus diseases (COVID-19) in China. Zhonghua Liu Xing Bing Xue Za Zhi 2020;41:145-51.

12. Chen N, Zhou M, Dong X, et al. Epidemiological and clinical characteristics of 99 cases of 2019 novel coronavirus pneumonia in Wuhan, China: a descriptive study. Lancet 2020;395:507-13.

13. Guan WJ, Ni ZY, Hu Y. Clinical characteristics of 2019 novel coronavirus infection in China. $\mathrm{N}$ Engl J Med 2020;382:1708-20.

14. Driggin E, Madhavan MV, Bikdeli B, et al. Cardiovascular considerations for patients, health care workers, and health systems during the COVID-19 pandemic. J Am Coll Cardiol 2020;75:2352-71.

15. Hammadah M, Sullivan S, Pearce B, et al. Inflammatory response to mental stress and mental stress induced myocardial ischemia. Brain Behav Immun 2018;68:90-7.

16. $\mathrm{Xu} X$, Chen $\mathrm{P}, \mathrm{Wang} \mathrm{J}$, et al. Evolution of the novel coronavirus from the ongoing Wuhan outbreak and modeling of its spike protein for risk of human

Cite this article as: Fan ZX, Yang J, Zhang J, He C, Wu H, Yang CJ, Zheng T, Ma C, Xiang ZJ, Zhai YH, Jiang J, Qiu SQ, Yang J. Analysis of influencing factors related to elevated serum troponin I level for COVID-19 patients in Yichang, China. Cardiovasc Diagn Ther 2020;10(4):678-686. doi: 10.21037/cdt20-510 transmission. Sci China Life Sci 2020;63:457-60.

17. Sodhi CP, Wohlford-Lenane C, Yamaguchi Y, et al. Attenuation of pulmonary ACE2 activity impairs inactivation of des-Arg9 bradykinin/BKB1R axis and facilitates LPS-induced neutrophil infiltration. Am J Physiol Lung Cell Mol Physiol 2018;314:L17-31.

18. Xu Z, Shi L, Wang YJ, et al. Pathological findings of COVID-19 associated with acute respiratory distress syndrome. Lancet Respir Med 2020;8:420-2.

19. Saetre T, Lindgaard AK, Lyberg T. Systemic activation of coagulation and fibrynolysis in a porcine model of serogroup A streptococcal shock. Blood Coagul Fibrinolysis 2000;11:433-8.

20. Tamis-Holland JE, Jneid H, Reynolds HR, et al. Contemporary diagnosis and management of patients with myocardial infarction in the absence of obstructive coronary artery disease: a scientific statement from the American heart association. Circulation 2019;139:e891-908.

21. Ordway GA, Garry DJ. Myoglobin: an essential hemoprotein in striated muscle. J Exp Biol 2004;207:3441-6.

22. Jialal I, Sokoll LJ. Clinical utility of lactate dehydrogenase: a historical perspective. Am J Clin Pathol 2015;143:158-9. 